\title{
CARACTERÍSTICAS DE ENFERMEDAD PULMONAR OBSTRUCTIVA CRÓNICA POR CONSUMO DE TABACO EN PACIENTES DE SERVICIOS DE NEUMOLOGÍA DE LIMA Y CALLAO
}

\author{
Alfredo G. Guerreros ${ }^{1,2, a}$, Eneyda G. Llerena ${ }^{3, a}$, Alberto Matsuno $^{4, a}$, Rolando Estrella ${ }^{5, a}$, Alejandro Peña ${ }^{6, a}$, \\ Hernando Torres-Zevallos ${ }^{7, b}$
}

\begin{abstract}
RESUMEN
El objetivo del estudio fue describir las características clínicas de pacientes con enfermedad pulmonar obstructiva crónica (EPOC), atendidos en los servicios de Neumología de centros especializados de Lima y Callao. Se realizó un estudio transversal en 196 pacientes, con una mediana de edad de 69 años, rango intercuartil 63-75 años. El 31,1\% de los pacientes se encontró en el grupo GOLD 1/leve (VEF1 $\geq 80 \%$ ), el 41,8 \% en el grupo GOLD 2/moderado (50 \% $\leq$ VEF1<80 \%), el $22,5 \%$ en el grupo GOLD 3/severo (30\% $\%$ VEF1<50 \%), y el 4,6 \% en el grupo GOLD 4/muy severo (VEF1<30 \%). El 93,9 \% usaba algún tipo de medicación para EPOC, la terapia más usada fue la combinación de beta agonistas de acción larga/corticoides inhalados (LABA/ICS) con 31,1\%. Se ha obtenido una primera caracterización de pacientes con EPOC atendidos en estos centros. Es imprescindible complementar lo encontrado con estudios longitudinales.
\end{abstract}

Palabras clave: Exacerbación; Altitud; Biomasa; Tuberculosis (fuente: DeCS BIREME).

\section{CHARACTERISTICS OF CHRONIC OBSTRUCTIVE PULMONARY DISEASE DUE TO TOBACCO CONSUMPTION IN PNEUMOLOGY PATIENTS IN LIMA AND CALLAO}

\begin{abstract}
The aim of the study was to describe the clinical characteristics of patients with COPD receiving treatment at the pneumology units of specialized care centers in Lima and Callao. A cross-sectional study was performed on 196 patients, with a mean age of 69 years, interquartile range of $63-75$ years. Of these patients, $31.1 \%$ was classified in the GOLD $1 /$ mild group (FEV1 $\geq 80 \%$ ); $41.8 \%$ in the GOLD $2 /$ moderate group $(50 \% \leq F E V 1<80 \%$ ); $22.5 \%$ in the GOLD $3 /$ severe group $(30 \% \leq \mathrm{FEV} 1<50 \%)$, and $4.6 \%$ in the GOLD $4 /$ very severe group (FEV1 $<30 \%)$. A $93.9 \%$ of patients used some type of medication for COPD. The most-frequently used therapy was the combination of long-acting beta agonists and inhaled corticosteroids (LABA/ICS) $(31.1 \%)$. A preliminary characterization has been obtained for COPD patients treated in these centers, but it is essential to complement these findings with longitudinal studies.
\end{abstract}

Keywords: Exacerbation; Altitude; Biomass; Tuberculosis (source: MeSH NLM).

\section{INTRODUCCIÓN}

La enfermedad pulmonar obstructiva crónica (EPOC) es una de las principales causas de muerte en todo el mundo (1) y supone un elevado costo económico (2).
En Latinoamérica se han realizado esfuerzos por conocer cada vez más a fondo las características de esta patología, investigaciones epidemiológicas como el estudio Platino ${ }^{(3)}$, y el estudio Puma ${ }^{(4)}$, son muestra de ello. También se han

\footnotetext{
Servicio de Neumología, Clínica Internacional. Lima, Perú

Facultad de Medicina, Universidad Nacional Mayor de San Marcos. Lima, Perú

Servicio de Neumología, Hospital Nacional Daniel Alcides Carrión. Bellavista, Callao, Perú

Servicio de Neumología, Clínica San Pablo, Santiago de Surco. Lima, Perú

Servicios médicos respiratórios independientes, Clínica San Bernardo. Pueblo Libre, Lima, Perú

Servicio de Neumología, Instituto Nacional del Corazón. Jesús María, Lima, Perú

Escuela de Salud Pública y Administración, Carlos Vidal Layseca, Universidad Peruana Cayetano Heredia. San Martín De Porres, Lima, Perú

Médico especialista en Neumología; ${ }^{\mathrm{b}}$ médico especialista en Estadística

Recibido: 12/01/2018 Aprobado: 18/04/2018 En línea: 28/06/2018
}

Citar como: Guerreros AG, Llerena EG, Matsuno A, Estrella R, Peña A, Torres-Zevallos H. Características de enfermedad pulmonar obstructiva crónica por consumo de tabaco en pacientes de servicios de neumología de Lima y Callao. Rev Peru Med Exp Salud Publica. 2018;35(2):265-71. doi:10.17843/rpmesp.2018.352.3377. 
realizado estudios sobre EPOC relacionados a la altitud de las ciudades ${ }^{(7,8)}$. En Perú, se han realizado estudios epidemiológicos para determinar los factores de riesgo atribuibles, en poblaciones urbanas y rurales, y en regiones de gran altitud ${ }^{(7,8)}$; así como estudios de intervención en el humo de biomasa, por ser uno de los principales factores implicados ${ }^{(9)}$. Sin embargo, se carece de datos clínicos de pacientes que estén siendo tratados por EPOC.

Los aspectos clínicos de la EPOC comprenden el antecedente de consumo de tabaco $u$ otras exposiciones ambientales, comorbilidades, síntomas, terapéutica usada y pruebas de función pulmonar. Actualmente, la clasificación más objetiva de la EPOC se basa en los parámetros espirométricos de la clasificación GOLD por The Global Initiative for Chronic Obstructive Lung Disease, basado en los valores del VEF1 (volumen espiratorio forzado al primer segundo) porcentual ${ }^{(1)}$.

Este estudio tiene como objetivo describir las características clínicas de pacientes con EPOC por consumo de cigarro, según la clasificación GOLD, en servicios de Neumología de las ciudades de Lima y Callao, en Perú.

\section{EL ESTUDIO}

Estudio multicéntrico, de corte transversal, descriptivo. El plan inicial fue recopilar información de 300 pacientes de centros ubicados en Lima Metropolitana y en la Provincia Constitucional del Callao, en Perú. Esta cifra se basó en la cantidad de pacientes con este diagnóstico, atendidos entre todos los centros, durante el periodo de los doce meses previos al inicio del estudio. Participaron cuatro centros, un hospital de referencia nacional y tres clínicas privadas. A cada centro se le asignó equitativamente un número de pacientes a ingresar en el estudio.

Se incluyeron a pacientes que firmaron su consentimiento informado; pacientes con diagnóstico de EPOC de acuerdo con la iniciativa GOLD; hombre o mujer de 40 o más años de edad; fumador o exfumador con historia de consumo de cigarrillos (por lo menos diez paquetes al año); pacientes tratados regularmente en el servicio (por lo menos una asistencia en el último año) y tener una espirometría previa, con una antigüedad no superior a 12 meses, con valores aceptables de calidad del volumen espiratorio forzado en el primer segundo (FEV1), para lo cual fue necesario cumplir con los criterios de aceptabilidad y repetitividad de la Sociedad Americana de Tórax / Sociedad Europea Respiratoria (ATS/ERS por American Thoracic Society/ European Respiratory Society); esta debe incluir una prueba posbroncodilatador realizada después de diez o 15 minutos de haber inhalado $400 \mu \mathrm{g}$ de salbutamol mediante

\section{MENSAJES CLAVE}

Motivación para realizar el estudio. La EPOC es una de las principales causas de muerte en el mundo y uno de los principales antecedentes es el consumo de tabaco. En Perú se carece de datos clínicos de pacientes que estén siendo tratados por EPOC.

Principales hallazgos. Según la función pulmonar de los pacientes, el 31,1 \% tuvo una limitación de flujo leve, el 41,8 \% moderado, el $22,5 \%$ severo y el $4,6 \%$ muy severo. El $45,9 \%$ de pacientes tenían también hipertensión arterial y el 93,9\% de pacientes usaba por lo menos alguna medicación para la EPOC.

Implicancias. Los resultados nos brindan un acercamiento a la situación de la EPOC en Perú. Es importante conocer con detalle el perfil del paciente, con la finalidad de brindarle una mejor atención médica.

aerosol dosificador presurizado, por lo que la dosis debería de haber sido administrada durante los siguientes 30 minutos después de realizada la prueba de espirometría prebroncodilatador.

Se excluyeron a pacientes que tuvieron otra enfermedad pulmonar crónica, como fibrosis quística o enfermedad pulmonar restrictiva; a pacientes que presentaron alguna entidad clínica que le impida entregar información confiable, o que le impida comunicarse con el investigador. El enrolamiento de pacientes al estudio fue de forma consecutiva según acudían a sus respectivos controles.

Para la recolección de datos se realizaron reuniones de trabajo a fin de seleccionar la información a recolectar y unificar los criterios de algunas de las respuestas. Para los datos clínicos, se recogió información de las historias clínicas de los pacientes mediante un cuestionario elaborado para el estudio. La espirometría fue obtenida de la historia clínica de cada paciente, pasó por un revisor externo, un neumólogo con subespecialidad en Fisiología Respiratoria, quien tenía que aceptar o no la prueba.

Las variables consideradas fueron: edad (incluidos únicamente mayores de 18 años); índice de masa corporal; género (masculino o femenino); estado civil (si estaba o no casado al momento del estudio, se consideró conviviente como casado); la variable raza fue establecida a criterio del evaluador, se categorizó según la predominancia (asiático, blanco, nativo americano, negro, o mestizo); el antecedente de haber vivido en altitud (se consideró positivo a quienes vivieron por lo menos un año y a una altitud de $1000 \mathrm{msnm}$ o mayor); el antecedente de consumo de tabaco (se estableció en paquetes de cigarros al año); y el antecedente de 
exposición a humo de biomasa (por un periodo mayor o igual a 10 años). La presencia de comorbilidades: antecedente de tuberculosis pulmonar, presencia de neumonía luego del diagnóstico de EPOC, indicación de uso de oxígeno, vacuna antineumocócica en los cuatro años previos a la evaluación y vacuna contra la influenza en el año previo a la evaluación. Se recogieron datos sobre la presencia o ausencia de síntomas de la EPOC, asimismo cada paciente completó la prueba de evaluación de la EPOC (CAT por COPD Assessment Test), con puntajes mayores o iguales a diez, lo que implicaron un impacto clínico medio o alto.

Las exacerbaciones fueron categorizadas de acuerdo con la presencia de dos o más en los últimos doce meses, y si presentó por lo menos una hospitalización por EPOC en el mismo periodo. La adherencia al tratamiento se midió a través de autoreporte con base en la escala: todos los días indicados; la gran mayoría de días; cuando me acuerdo, pero no la mayoría de los días; sólo cuando me siento mal y casi nunca sigo las indicaciones (se consideró como adherente sólo a las dos primeras). La evaluación del grado de limitación del flujo aéreo por medio de espirometría, en base a los valores del VEF1 porcentual, se generaron cuatro categorías: GOLD 1: $\geq 80 \%$, GOLD 2: $50 \%$ - $79 \%$, GOLD 3: $30 \%$ - $49 \%$, y GOLD 4: <30 \%.

El plan de análisis estadístico constó de una primera fase que corresponde al procesamiento de datos. Posteriormente se siguió con el análisis univariado, las variables cuantitativas se resumen en mediana y rango intercuartílico, y porcentajes para las variables cualitativas. A fin de mostrar mayor detalle en el análisis, se disgregó la información en los cuatro grupos de la clasificación GOLD. Para el análisis entre los grupos de la clasificación GOLD se usó la prueba no paramétrica de Kruskal Wallis para las variables cuantitativas y Chi cuadrado de Pearson para las variables categóricas. La significancia estadística se estableció en $p<0,05$ para dos colas. Se utilizó el paquete estadístico STATA 14, Statistics Data Analysis, StataCorp, College Station, Texas, EE. UU.

El estudio contó con la aprobación del Comité Institucional de Ética en Investigación de la Universidad Peruana Cayetano Heredia, número de registro 64649. Todos los pacientes firmaron un consentimiento informado antes de participar en el estudio.

\section{HALLAZGOS}

Duranteel periodo2015a2017 se incluyerona 196 pacientes, provenientes de tres centros de Lima Metropolitana y uno de la Provincia Constitucional del Callao, en Perú (Figura 1). La Tabla 1 nos muestra las características demográficas.
El 31,1\% (61) de los pacientes se encontró en el grupo GOLD 1/leve; el 41,8 \% (82) en el grupo GOLD 2/moderado; el 22,5 \% (44) en el grupo GOLD 3/severo y el 4,6 \% (9) en el grupo GOLD 4/muy severo.

En la Tabla 2 se muestran las características clinicas relacionados a la EPOC. En la Tabla 3 se describe el uso de medicación para la EPOC, se describe el uso de medicación de rescate, cualquier combinación de medicación de mantenimiento y autoreporte de adherencia. Al momento

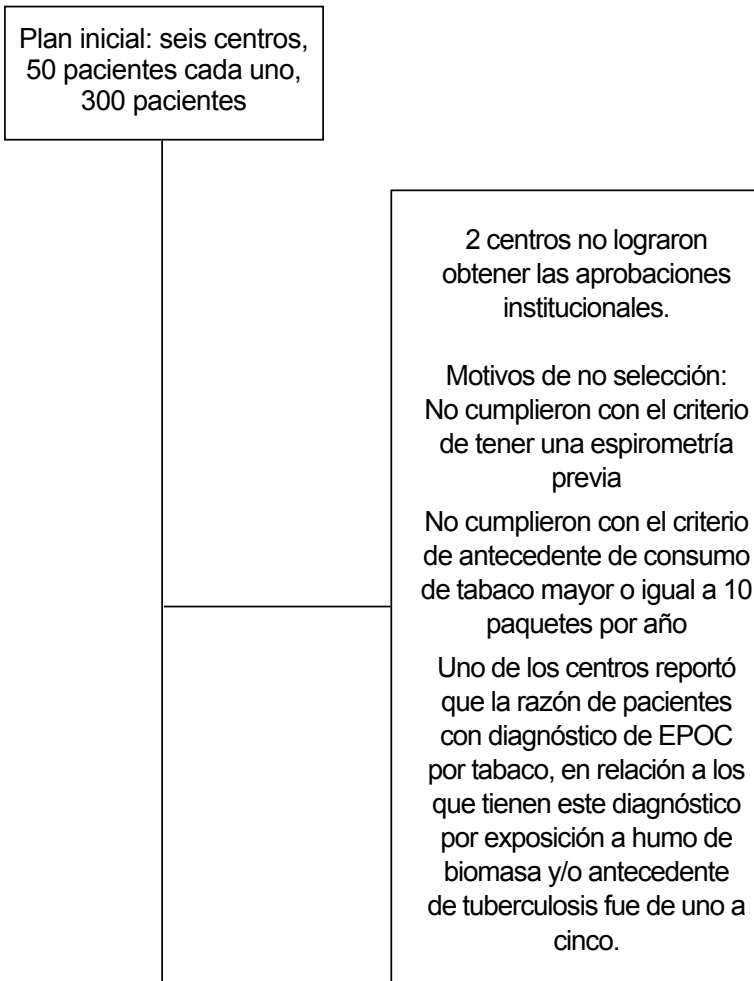

4 centros, 206 pacientes seleccionados*

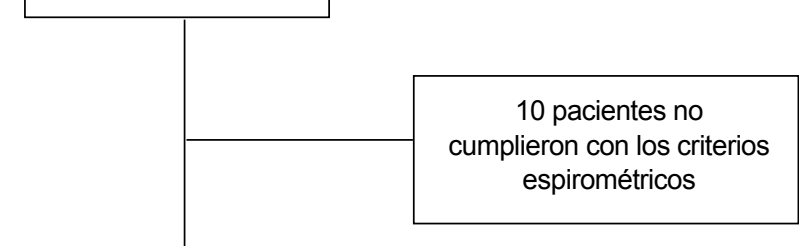

196 pacientes incluidos en el análisis

${ }^{*}$ ) Se respetó estrictamente los tiempos asignados para el periodo de reclutamiento de pacientes.

Figura 1. Selección de participantes del estudio 
Tabla 1. Características demográficas de pacientes con Enfermedad Pulmonar Obstructiva Crónica por consumo de tabaco atendidos en servicios de neumología (Lima y Callao)

\begin{tabular}{|c|c|c|c|c|c|c|}
\hline Características & Total $(n=196)$ & GOLD 1 (n=61) & GOLD 2 (n=82) & GOLD $3(n=44)$ & GOLD 4 (n=9) & Valor de $p$ * \\
\hline Edad, mediana (RIC) & $69(63-75)$ & $68(62-75)$ & $68(63-75)$ & $71,5(64,5-79,5)$ & $72(66-75)$ & $0,338^{\dagger}$ \\
\hline IMC, mediana (RIC) & $25,0(23,0-28,1)$ & $25,4(22,5-27,9)$ & $25,2(23,7-28,2)$ & $24,8(22,6-29,1)$ & $23,2(17,8-24,2)$ & $0,071^{\dagger}$ \\
\hline Femenino, n (\%) & $51(26,0)$ & $12(19,7)$ & $22(26,8)$ & $14(31,8)$ & $3(33,3)$ & 0,508 \\
\hline Casado, n (\%) & $120(61,2)$ & $39(63,9)$ & $51(62,2)$ & $25(56,8)$ & $5(55,6)$ & 0,873 \\
\hline Raza, n (\%) & & & & & & 0,571 \\
\hline Asiático & $28(14,3)$ & $7(11,5)$ & $13(15,9)$ & $6(13,6)$ & $2(22,2)$ & \\
\hline Blanco & $56(28,6)$ & $15(24,6)$ & $24(29,3)$ & $13(29,6)$ & $4(44,5)$ & \\
\hline Mestizo & $32(16,3)$ & $11(18,0)$ & $15(18,3)$ & $4(9,1)$ & $2(22,2)$ & \\
\hline Nativo & $63(32,1)$ & $19(31,1)$ & $25(30,5)$ & $18(40,9)$ & $1(11,1)$ & \\
\hline Negro & $17(8,7)$ & $9(14,8)$ & $5(6,1)$ & $3(6,8)$ & $0(0,0)$ & \\
\hline Vivió en altitud, $n$ (\%) & $38(19,4)$ & $17(27,9)$ & $9(10,9)$ & $11(25,0)$ & $1(11,1)$ & 0,050 \\
\hline
\end{tabular}

RIC: rango intercuartílico, IMC: Índice de masa corporal

GOLD 1: FEV1 $\geq 80 \%$ (volumen espiratorio forzado en el primer segundo mayor de $80 \%$ )

GOLD 2: $50 \% \leq F E V 1<80 \%$ (volumen espiratorio forzado en el primer segundo entre $50 \%$ y $80 \%$ )

GOLD 3: $30 \% \leq F E V 1<50 \%$ (volumen espiratorio forzado en el primer segundo entre $30 \%$ y $50 \%$ )

GOLD 4: FEV1<30\% (volumen espiratorio forzado en el primer segundo menor de $30 \%$ )

* Prueba de Chi cuadrado, ${ }^{\dagger}$ Prueba de Kruskal Wallis

del estudio la gran mayoría de pacientes usaba terapia de mantenimiento $(74 \%, 145)$, en forma de monoterapia la usaba el 7,7 \% (15), terapia doble el 39,3\% (77), y terapia triple el $17,8 \%$ (35).

Se encontraron diferencias estadísticamente significativas en relación a la severidad de la EPOC según grupos GOLD, para los variables paquetes año, uso de oxígeno domiciliario, y uso de cualquier terapia para EPOC.

\section{DISCUSIÓN}

Se describen los aspectos demográficos, y clínicos de pacientes atendidos ambulatoria y regularmente en servicios especializados de neumología de Lima y Callao. Los aspectos relacionados a pacientes con EPOC como sintomatología, consumo de tabaco, comorbilidades (10), exacerbaciones y uso de medicación ${ }^{(11)}$, son similares a lo reportado a nivel mundial ${ }^{(12)}$.

Fue muy llamativo el uso de corticoides inhalados como medicación de mantenimiento en un gran porcentaje de pacientes, contrariamente a lo recomendado en la iniciativa GOLD. Sin embargo, esta tendencia está presente en otros países ${ }^{(13,14)}$. Se suele reportar problemas con la adherencia a la medicación para pacientes con EPOC ${ }^{(15)}$, en nuestro caso encontramos un alto porcentaje de adherencia a la terapia, esto puede explicarse por el tipo de pacientes estudiados, con un gran porcentaje de disnea, o puede estar sobreestimado al ser autoreporte.

Las limitaciones del presente estudio están determinadas por su diseño, se trata de una muestra no representativa al estar circunscrito al ámbito hospitalario y de atención secundaria (pacientes en tratamiento por neumólogos), así también el muestreo fue no aleatorio. Dentro de los aspectos técnicos, otra limitación fue la utilización por cada centro de su propio espirómetro, sin embargo, se pretendió subsanar este problema con un revisor externo. A pesar de estas limitaciones, esta investigación presenta un primer acercamiento de lo que actualmente ocurre con los pacientes con EPOC por tabaquismo, así como ser el punto de partida para proponer nuevos estudios longitudinales como los realizados en otros países ${ }^{(16,17,18)}$, con especial atención en la población femenina por el gran infradiagnóstico existente ${ }^{(19)}$, y sobre efectos de la polución ${ }^{(20)}$.

En conclusión, hemos obtenido una primera caracterización de pacientes con EPOC atendidos en centros especializados de Lima y Callao de Perú. La procedencia, antecedentes, patologías y hábitos (humo de biomasa) pueden influir en la enfermedad. De lo hallado nos surgen muchas preguntas que deben transformarse, obligatoriamente, en nuevas investigaciones. 
Tabla 2. Características clínicas de pacientes con Enfermedad Pulmonar Obstructiva Crónica por consumo de tabaco atendidos en servicios de neumología (Lima y Callao)

\begin{tabular}{|c|c|c|c|c|c|c|}
\hline Características clínicas & $\begin{array}{c}\text { Total } \\
(n=196)\end{array}$ & $\begin{array}{l}\text { GOLD } 1 \\
(n=61)\end{array}$ & $\begin{array}{l}\text { GOLD } 2 \\
(n=82)\end{array}$ & $\begin{array}{l}\text { GOLD } 3 \\
(n=44)\end{array}$ & $\begin{array}{c}\text { GOLD } 4 \\
(n=9)\end{array}$ & $\begin{array}{l}\text { Valor } \\
\text { de } p \text { * }\end{array}$ \\
\hline \multicolumn{7}{|l|}{ Comorbilidades, n (\%) } \\
\hline Hipertensión & $97(49,5)$ & $29(47,6)$ & $40(48,8)$ & $25(56,8)$ & $3(33,3)$ & 0,574 \\
\hline Diabetes mellitus & $21(10,7)$ & $7(11,5)$ & $9(11,0)$ & $5(11,4)$ & $0(0,0)$ & 0,767 \\
\hline Osteoporosis & $12(6,1)$ & $2(3,3)$ & $6(7,3)$ & $4(9,1)$ & $0(0,0)$ & 0,508 \\
\hline Dislipidemia & $21(10,7)$ & $3(4,9)$ & $12(14,6)$ & $3(6,8)$ & $3(33,3)$ & 0,030 \\
\hline Depresión & $30(15,3)$ & $9(14,8)$ & $16(19,5)$ & $5(11,4)$ & $0(0,0)$ & 0,349 \\
\hline Ansiedad & $30(15,3)$ & $10(16,4)$ & $14(17,1)$ & $6(13,6)$ & $0(0,0)$ & 0,578 \\
\hline ERGE & $17(8,7)$ & $8(13,1)$ & $5(6,1)$ & $3(6,8)$ & $1(11,1)$ & 0,482 \\
\hline Consumo de tabaco, mediana $(\mathrm{RIC}) \S$ & $30(20-47,5)$ & $25,5(18-40)$ & $30(19,8-50)$ & $30(22,5-50,5)$ & $47(40-60)$ & $0,013^{\dagger}$ \\
\hline Fumador actual, n (\%) & $48(24,5)$ & $14(22,9)$ & $22(26,8)$ & $10(22,7)$ & $2(22,2)$ & 0,936 \\
\hline Biomasa, n (\%) & $31(15,8)$ & $14(22,9)$ & $9(11,0)$ & $8(18,2)$ & $0(0,0)$ & 0,130 \\
\hline Antecedente de tuberculosis, n (\%) & $46(23,5)$ & $16(26,2)$ & $18(22,0)$ & $10(22,7)$ & $2(22,2)$ & 0,943 \\
\hline Uso de oxígeno, n (\%) & $12(6,1)$ & $1(1,6)$ & $2(2,4)$ & $5(11,4)$ & $4(44,4)$ & $<0,001$ \\
\hline \multicolumn{7}{|l|}{ Vacunas, n (\%) } \\
\hline Influenza & $126(64,3)$ & $42(68,9)$ & $46(56,1)$ & $32(72,7)$ & $6(66,7)$ & 0,227 \\
\hline Antineumocócica & $43(21,9)$ & $14(22,9)$ & $14(17,1)$ & $12(27,3)$ & $3(33,3)$ & 0,460 \\
\hline \multicolumn{7}{|l|}{ Síntomas, n (\%) } \\
\hline Tos & $186(94,9)$ & $60(98,4)$ & $77(93,9)$ & $40(90,9)$ & $9(100,0)$ & 0,307 \\
\hline Flema & $181(92,4)$ & $55(90,2)$ & $76(92,7)$ & $41(93,2)$ & $9(100,0)$ & 0,750 \\
\hline Dolor de pecho & $139(70,9)$ & $44(72,1)$ & $55(67,1)$ & $32(72,7)$ & $8(89,9)$ & 0,550 \\
\hline Disnea & $182(92,9)$ & $54(88,5)$ & $76(92,7)$ & $43(97,7)$ & $9(100,0)$ & 0,262 \\
\hline CAT $\geq 10, n(\%)$ & $162(82,7)$ & $50(82,0)$ & $65(79,3)$ & $38(86,4)$ & $9(100,0)$ & 0,394 \\
\hline Puntaje CAT, mediana (RIC) & $20(12-28)$ & $21(12-32)$ & $17,5(10-26)$ & $20,5(13-26)$ & $23(22-24)$ & $0,133^{\dagger}$ \\
\hline Exacerbaciones $\geq 2, \mathrm{n}(\%)^{\ddagger}$ & $47(24,0)$ & $15(24,6)$ & $15(18,3)$ & $14(31,8)$ & $3(33,3)$ & 0,336 \\
\hline Hospitalizaciones $\geq 1, \mathrm{n}(\%)^{\ddagger}$ & $13(6,6)$ & $5(8,2)$ & $2(2,4)$ & $5(11,4)$ & $1(11,1)$ & 0,217 \\
\hline Neumonía, $\mathrm{n}(\%) \ddagger$ & $16(8,2)$ & $3(4,9)$ & $8(9,8)$ & $4(9,1)$ & $1(11,1)$ & 0,732 \\
\hline
\end{tabular}

ERGE: enfermedad por reflujo gastroesofágico, CAT: Prueba de evaluación de EPOC (COPD Assessment Test).

GOLD 1: FEV1 $\geq 80 \%$ (volumen espiratorio forzado en el primer segundo mayor de $80 \%$ )

GOLD 2: $50 \% \leq \mathrm{FEV} 1<80 \%$ (volumen espiratorio forzado en el primer segundo entre $50 \%$ y $80 \%$ )

GOLD 3: $30 \% \leq \mathrm{FEV} 1<50 \%$ (volumen espiratorio forzado en el primer segundo entre $30 \%$ y $50 \%$ )

GOLD 4: FEV1<30\% (volumen espiratorio forzado en el primer segundo menor de $30 \%$ )

* Prueba de Kruskal Wallis

${ }^{\dagger}$ Prueba de Chi cuadrado

₹ Los casos se presentaron dentro de los últimos 12 meses

$\S$ Consumo de paquetes de cigarros al año

Contribuciones de autoría: AGG y HT han participado en el diseño y concepción del estudio. AGG, EGL, AM, RE y HT han participado en el reclutamiento de pacientes. HTZ ha participado en el análisis estadístico. AP ha participado como revisor de las pruebas funcionales (espirometrías) de todos los pacientes. AGG ha obtenido el financiamiento para el presente estudio. Todos los autores han participado en la redacción y aprobación final del manuscrito. Novartis no ha participado en el diseño, análisis de datos, ni en la presentación de resultados estudio. 
Tabla 3. Medicación usada para Enfermedad Pulmonar Obstructiva Crónica por consumo de tabaco en servicios de neumología (Lima y Callao)

\begin{tabular}{lcccccc}
\hline Medicación, $\mathbf{n}(\mathbf{\%})$ & $\begin{array}{c}\text { Total } \\
(\mathbf{n = 1 9 6 )}\end{array}$ & $\begin{array}{c}\text { GOLD 1 } \\
(\mathbf{n = 6 1 )}\end{array}$ & $\begin{array}{c}\text { GOLD 2 } \\
(\mathbf{n = 8 2})\end{array}$ & $\begin{array}{c}\text { GOLD 3 } \\
(\mathbf{n = 4 4 )}\end{array}$ & $\begin{array}{c}\text { GOLD 4 } \\
(\mathbf{n = 9})\end{array}$ & Valor de $\mathbf{p}$ * \\
\hline SABA o SAMA & $174(88,8)$ & $51(83,6)$ & $74(90,2)$ & $40(90,9)$ & $9(100,0)$ & 0,369 \\
SABA o SAMA solamente & $34(17,4)$ & $14(22,9)$ & $16(19,5)$ & $3(6,8)$ & $1(11,1)$ & 0,154 \\
Cualquier terapia de mantenimiento & $145(74,0)$ & $36(59,0)$ & $62(75,6)$ & $39(88,6)$ & $8(88,9)$ & 0,004 \\
Cualquier terapia & $184(93,9)$ & $53(86,9)$ & $79(96,3)$ & $43(97,7)$ & $9(100,0)$ & 0,051 \\
SABA & $168(85,7)$ & $48(78,7)$ & $72(87,8)$ & $40(90,9)$ & $8(88,9)$ & 0,284 \\
SAMA & $34(17,4)$ & $14(22,9)$ & $9(11,0)$ & $10(22,7)$ & $1(11,1)$ & 0,188 \\
LABA monoterapia & $6(3,1)$ & $3(4,9)$ & $2(2,4)$ & $1(2,3)$ & $0(0,0)$ & 0,755 \\
LAMA monoterapia & $9(4,6)$ & $4(6,6)$ & $4(4,9)$ & $1(2,3)$ & $0(0,0)$ & 0,676 \\
ICS monoterapia & $18(9,2)$ & $8(13,1)$ & $8(9,8)$ & $2(4,6)$ & $0(0,0)$ & 0,361 \\
LABA/ICS & $61(31,1)$ & $13(21,3)$ & $27(32,9)$ & $19(43,2)$ & $2(22,2)$ & 0,103 \\
LABA+ICS & $6(3,1)$ & $1(1,6)$ & $3(3,7)$ & $2(4,6)$ & $0(0,0)$ & 0,771 \\
LABA/LAMA & $1(0,5)$ & $0(0,0)$ & $0(0,0)$ & $1(2,3)$ & $0(0,0)$ & 0,324 \\
LABA+LAMA & $7(3,6)$ & $0(0,0)$ & $5(6,1)$ & $2(4,6)$ & $0(0,0)$ & 0,237 \\
LAMA+ICS & $2(1,0)$ & $2(3,3)$ & $0(0,0)$ & $0(0,0)$ & $0(0,0)$ & 0,215 \\
LABA/ICS+LAMA & $33(16,8)$ & $5(8,2)$ & $13(15,9)$ & $10(22,7)$ & $5(55,6)$ & 0,003 \\
LABA/LAMA+ICS & $1(0,5)$ & $0(0,0)$ & $0(0,0)$ & $1(2,3)$ & $0(0,0)$ & 0,324 \\
LABA+LAMA+ICS & $1(0,5)$ & $0(0,0)$ & $0(0,0)$ & $0(0,0)$ & $1(11,1)$ & $<0,001$ \\
\hline Otra terapia & $24(12,2)$ & $9(14,8)$ & $10(12,2)$ & $9,1 \%(4)$ & $1(11,1)$ & 0,855 \\
Adherencia & $168(85,7)$ & $46(75,4)$ & $75(91,5)$ & $86,4 \%(38)$ & $9(100,0)$ & 0,029 \\
\hline
\end{tabular}

SABA: Beta agonistas de acción corta (Short-acting beta agonist), SAMA: Antimuscarínicos de acción corta (Short-acting muscarinic antagonist), LABA: Beta agonistas de acción larga (Long-acting beta agonists), LAMA: Antmuscarínicos de acción larga (Long-acting muscarinic antagonists), Cl: corticoides inhalados. Otra terapia: incluye xantinas, corticoides orales o antibióticos. El símbolo barra oblicua (/) denota medicación manufacturada de forma de combinación. El signo más $(+)$ denota el uso de dos más medicamentos.

GOLD 1: FEV1 $\geq 80 \%$ (volumen espiratorio forzado en el primer segundo mayor de $80 \%$ )

GOLD 2: $50 \% \leq \mathrm{FEV} 1<80 \%$ (volumen espiratorio forzado en el primer segundo entre $50 \%$ y $80 \%$ )

GOLD 3: $30 \% \leq F E V 1<50 \%$ (volumen espiratorio forzado en el primer segundo entre $30 \%$ y $50 \%$ )

GOLD 4: FEV1<30\% (volumen espiratorio forzado en el primer segundo menor de $30 \%$ )

* Prueba de Chi cuadrado

Fuentes de financiamiento: El presente trabajo ha sido financiado por Novartis Biosciences. Concurso regional de estudios iniciados por el investigador para América Latina.

\section{Conflicto de intereses: Ninguno}

\section{REFERENCIAS BIBLIOGRÁFICAS}

1. Vogelmeier CF, Criner GJ, Martinez FJ, Anzuelo A, Barnes PJ, Bourbeau J; et al. Global strategy for the diagnosis, management, and prevention of chronic obstructive pulmonary disease 2017 report: GOLD executive summary. Am J Respir Crit Care Med. 2017;195(5):557582. doi: 10.1164/rccm.201701-0218PP.

2. Pasquale MK, Sun SX, Song F, Hartnett HJ, Stemkowski SA. Impact of exacerbations on health care cost and resource utilization in chronic obstructive pulmonary disease patients with chronic bronchitis from a predominantly Medicare population. Int J Chron Obstruct Pulmon Dis.
2012;7:757-64. doi: 10.2147/COPD. S36997.

3. Menezes AM, Perez-Padilla R, Jardim JR Muino A, Lopez MV, Valdivia G; et al. Chronic obstructive pulmonary disease in five Latin American cities (the PLATINO study): a prevalence study. Lancet. 2005;366(9500):1875-81. doi: 10.1016/ S0140-6736(05)67632-5.

4. Schiavi E, Stirbulov R, Hernández Vecino R, Mercurio S, Di Boscio V; Puma Team. COPD screening in primary care in four Latin American countries: methodology of the PUMA Study. Arch Bronconeumol. 2014;50(11):469-74. doi: 10.1016/j. arbres.2014.03.006.
5. Caballero A, Torres-Duque CA, Jaramillo C, Bolívar F, Sanabria F, Osorio P; et al. Prevalence of COPD in five $\mathrm{Co}$ lombian cities situated at low, medium, and high altitude (PREPOCOL study). Chest. 2008;133(2):343-9. doi:10.1378/ chest.07-1361.

6. Horner A, Soriano JB, Puhan MA, Studnicka M, Kaiser B, Vanfleteren LEGW; et al. Altitude and COPD prevalence: analysis of the PREPOCOL-PLATINO-BOLD-EPI-SCAN study. Respir Res. 2017;18(1):162. doi: 10.1186/ s12931-017-0643-5.

7. Jaganath D, Miranda JJ, Gilman RH, Wise RA, Diette GB, Miele CH; et al. Prevalence 
of chronic obstructive pulmonary disease and variation in risk factors across four geographically diverse resource-limited settings in Peru. Respir Res. 2015;16:40. doi: 10.1186/s12931-015-0198-2.

8. Miele $\mathrm{CH}$, Jaganath $\mathrm{D}$, Miranda JJ, Bernabe-Ortiz A, Gilman RH, Johnson $\mathrm{CM}$; et al. Urbanization and daily exposure to biomass fuel smoke both contribute to chronic bronchitis risk in a population with low prevalence of daily tobacco smoking. COPD. 2016;13(2):186-95. doi: $10.3109 / 15412555.2015$.

9. Accinelli R. Yshii C, Córdova E, SánchezSierra M, Pantoja C, Carbajal J. Efectos de los combustibles de biomasas en el aparato respiratorio: impacto del cambio a cocinas con diseño mejorado. Enf Tórax. 2004; 48(2):138-144.

10. Smith MC, Wrobel JP. Epidemiology and clinical impact of major comorbidities in patients with COPD. Int J Chron Obstruct Pulmon Dis. 2014;9:871-88. doi: 10.2147/COPD.S49621.

11. Montes de Oca M, Tálamo C,Perez-Padilla R, Lopez MV, Muiño A, Jardim JR; et al. Use of respiratory medication in five Latin American cities: The PLATINO study. Pulm Pharmacol Ther. 2008;21(5):78893. doi: 10.1016/j.pupt.2008.06.003.

12. Viejo-Bañuelos JL, Pueyo-Bastida A, Fueyo-Rodríguez A. Characteristics of outpatients with COPD in daily practice: The E4 Spanish project. Respir Med. 2006;100(12):2137-43.doi: $\quad 10.1016 / j$. rmed.2006.03.043.

13. Cosio BG, Soriano JB, López-Campos JL, Calle-Rubio M, Soler-Cataluna JJ, de-Torres JP; et al. Defining the asthmaCOPD overlap syndrome in a COPD cohort. Chest. 2016;149(1):45-52. doi: 10.1378/chest.15-1055.

14. Ding B, Small M. Treatment trends in patients with asthma-COPD overlap syndrome in a COPD cohort: findings from a real-world surveyInt J Chron Obstruct Pulmon Dis. 2017;12:1753-1763. doi: 10.2147/COPD.S136314.

15. Koehorst-ter Huurne K, Movig K, van der Valk P, van der Palen J, BrusseKeizer M. Differences in adherence to common inhaled medications in COPD. COPD. 2015;12(6):643-8. doi: $10.3109 / 15412555.2014 .995292$.

16. Ancochea J, Badiola C, Duran-Tauleria E, Garcia Rio F, Miravitlles M, Muñoz L; et al. Estudio EPI-SCAN: resumen del protocolo de un estudio para estimar la prevalencia de EPOC en personas de 40 a 80 años en España. Arch Bronconeumol. 2009;45(1):41-7. doi: 10.1016/j. arbres.2008.06.001.

17. Soriano JB, Ancochea J, Miravitlles M, García-Río F, Duran-Tauleria E, Muñoz L; et al. Recent trends in COPD prevalence in Spain: a repeated cross-sectional survey 1997-2007. Eur Respir J. 2010;36(4):75865. doi: 10.1183/09031936.00138409.
18. Menezes AM, Muiño A, López-Varela MV, Valdivia G, Lisboa C, Jardim JR; et al. A population-based cohort study on chronic obstructive pulmonary disease in Latin America: methods and preliminary results. The PLATINO Study Phase II. Arch Bronconeumol. 2014;50(1):10-7. doi: 10.1016/j.arbres.2013.07.014.

19. Ancochea J, Miravitlles M, García-Río F, Muñoz L, Sánchez G, Sobradillo V; et al. Underdiagnosis of chronic obstructive pulmonary disease in women: quantification of the problem, determinants and proposed actions. Arch Bronconeumol. 2013;49(6):223-9. doi: 10.1016/j.arbres.2012.11.010.

20. Eisner MD, Anthonisen N, Coultas D, Kuenzli N, Perez-Padilla R, Postma D; et al. An official American Thoracic Society public policy statement: Novel risk factors and the global burden of chronic obstructive pulmonary disease. Am J Respir Crit Care Med. 2010;182(5):693-718. doi: 10.1164/rccm.200811-1757ST.

Correspondencia: Hernando Torres Zevallos Correo electrónico:mdhertz@hotmail.com, hernando. torresz@upch.pe 